\title{
Chapter 12 \\ Model Predictive Control of Water Networks Considering Flow
}

\author{
Gabriela Cembrano, Vicenç Puig, Carlos Ocampo-Martínez, Meritxell \\ Minoves and Ramon Creus
}

\subsection{Introduction}

Decision support systems provide useful guidance for operators in complex networks, where resource management best actions are not intuitive. Optimization and optimal control techniques provide an important contribution to a smart management strategy computation for drinking-water networks (DWNs) (see [1-3]). Similarly, problems related to modelling and control of water supply, transport and distribution systems have been an object of important research efforts during the last few years (see, e.g., [4-7].

In general, DWNs contain multiple tanks, pumping stations, valves, water sources (superficial and underground) and sectors of consumer demand. Operational control of DWNs using optimal control techniques has been largely investigated (see [5]). This chapter proposes the use of model predictive control (MPC) techniques to generate flow control strategies in a transport network, delivering water from the drinkingwater treatment plants to the consumer areas to meet future demands. Set points for pumps and valves are computed by optimizing a performance index expressing operational goals such as economic cost, safety water storage and smoothness in flow control actions. The main point is to highlight the advantages of using optimizationbased control techniques, such as MPC, to improve the performance of a water transport network, taking into account their large-scale nature (in terms of number of dynamic elements and decision variables), the nature of the desired control objectives

G. Cembrano $(\varangle) \cdot$ C. Ocampo-Martínez

Institut de Robòtica i Informàtica Industrial, CSIC-UPC, Barcelona, Spain

e-mail: gcembrano@iri.upc.edu

M. Minoves $\cdot$ R. Creus

Aguas de Barcelona (AGBAR), Barcelona, Spain

V. Puig

Research Center "Supervision, Safety and Automatic Control" (CS2AC-UPC), Terrassa, Spain 
and the type and behaviour of the system disturbances (drinking-water demands). The developed control strategies have been tested on the drinking-water transport network of Barcelona.

\subsection{Problem Statement}

\subsubsection{Operational Control of Water Networks}

Complex nonlinear models are very useful for offline operations (for instance, calibration and simulation). Detailed mathematical representations such as the pressureflow models for DWNs allow the simulation of those systems with enough accuracy to observe specific phenomena, useful for design and investment planning. However, for online computation purposes such as those related to global management, a simpler and control-oriented model structure must be conveniently selected. This simplified model includes the following features:

(i) Representativeness of the main network dynamics: It must provide an evaluation of the main representative hydrological/hydraulic variables of the network and their response to control actions at the actuators.

(ii) Simplicity, expandability, flexibility and speed: It must use the simplest approach capable of achieving the given purposes, allowing very easily to expand and/or modify the modelled portion of the network.

(iii) Amenability to online calibration and optimization: This modelling approach must be easily calibrated online using data from the telemetry system and embedded in an optimization problem to achieve the network management objectives.

Figure 12.1, adapted from [8, 9], shows a hierarchical structure for a real-time control (RTC) water system. There, the MPC, as the global control law, determines the references (set points) for the local controllers placed at different elements of the networked system. These references are computed according to the measurements taken from sensors distributed around the network. The management level provides the MPC with its operational objectives, which are reflected in the controller design as the performance indices to be enhanced, which can be either minimized or maximized, depending on the case. Finally, water system control requires the use of a supervisory system to monitor the performance of the different control elements in the network (sensors and actuators) and to take appropriate correcting actions in the case where a malfunction is detected, to achieve a proper fault-tolerant control [10].

In most water networks, the operational control is managed by the operators from the telecontrol centre using a SCADA (supervisory control and data acquisition) system. Operators are in charge of supervising the network status using the telemetry system and providing the set points for the local controllers, which are typically based on PID algorithms. The main goal of the operational control of water networks is 


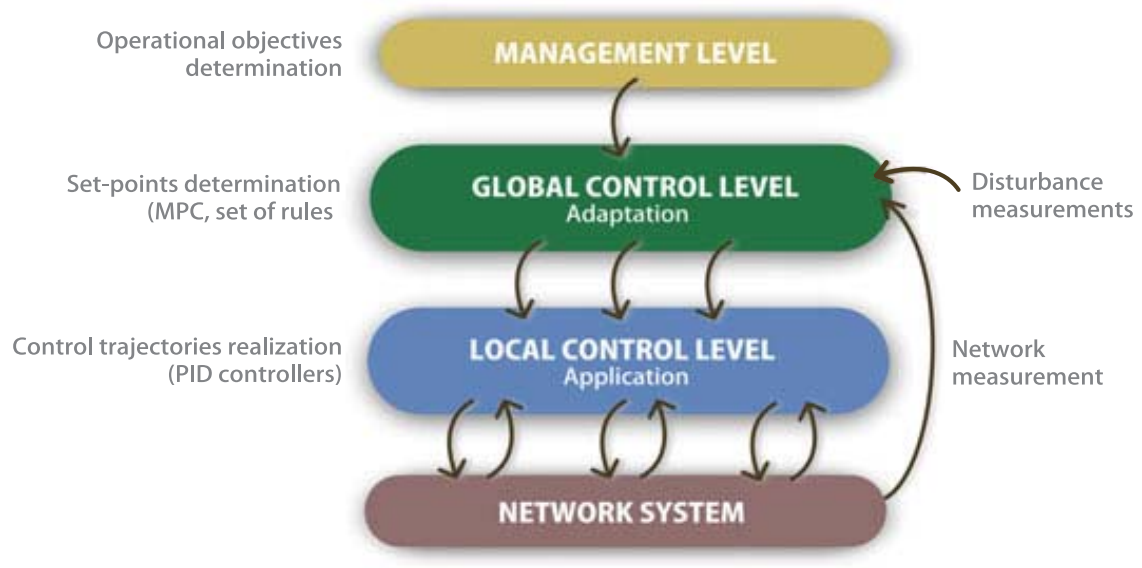

Fig. 12.1 Hierarchical structure for RTC system

to meet the demands at consumer sites, but at the same time with minimum costs of operation and guaranteeing pre-established volumes in tanks (to preserve the satisfaction of future demands) and smooth operation of actuators (valves and pumps) and production plants.

Water consumption in urban areas is usually managed on a daily basis, because water demand generally presents daily patterns and reasonably good hourly 24-hahead demand predictions may, in general, be available. Therefore, this horizon is appropriate for evaluating the effects of different control strategies on the water network, with respect to operational goals. However, other horizons may be more appropriate in specific utilities. The approach proposed here is based on demand satisfaction at the transport level, taking into account the supply conditions. For illustration, it uses - but is not restricted to - a 24-h horizon, with hourly sampling. When applied in real-time conditions, the computation of optimal strategies is updated, with new data from the water network, every hour with a sliding 24-h horizon.

At the supply water basin level, strategic planning deals with sustainable use of the water resources, seasonal variations in reservoirs and water levels, etc., so that planning horizon, sampling times and control time steps are usually much longer. In this work, the long-term planning objectives for the supplies are taken into account as bands of admissible requests from the supplies to the transport, production and distribution areas. These admissible bands define bounds on flow from reservoir, aquifer and river sources. Production plant limitations are also used, and these may vary according to weather-related factors, operational schedules and/or breakdowns. The computation of optimal strategies must take into account the dynamics of the complete water system and 24-h-ahead demand forecasts, availability predictions in supply reservoirs and aquifers, defined by long-term planning for sustainable use and predictions of production plant capacity and availability. Moreover, the telemetry system and operational database will provide the current state of the water system. 


\subsubsection{Operational Control of Water Network Using MPC}

Water networks are very complex multi-variable systems. MPC provides suitable techniques to implement the operational control of water systems to improve their performance, since it allows to compute optimal control strategies ahead in time for all the control elements $[11,12]$. Moreover, MPC allows taking into account physical and operational constraints, the multi-variable input and output nature, the demand forecasting requirement and complex multi-objective operational goals of water networks. The optimal strategies are computed by optimizing a mathematical function describing the operational goals in a given time horizon and using a representative model of the network dynamics, as well as demand forecasts.

\subsection{Proposed Approach}

The aim of using MPC techniques for controlling DWNs is to compute, ahead in time, the input actions to achieve the optimal performance of the network according to a given set of control goals. MPC strategies have some important features to deal with complex systems such as DWNs, namely the amenability to include disturbance forecasts, physical constraints and multi-variable system dynamics and objectives in a relatively simple way.

\subsubsection{Modelling}

Several modelling techniques dealing with DWNs have been presented in the literature (see, e.g., [5, 13]). Here, a control-oriented modelling approach that considers a flow model is outlined, which follows the principles presented by the authors in [6, 14, 15]. The extension to include the pressure model can be found in Chap. 13. A DWN generally contains a set of pressurized pipes, water tanks at different elevation and a number of pumping stations and valves to manage water flows, pressure and elevation in order to supply water to consumers.

The DWN model can be considered as composed of a set of constitutive elements, which are presented and discussed below. Figure 12.2 shows, in a small example, the interconnection of typical constitutive elements.

\subsubsection{Tanks}

Water tanks provide the entire DWN with the storage capacity of drinking water at appropriate elevation levels to ensure adequate water pressure service to consumers. The mass balance expression relating to the stored volume $v$ in the $n$th tank can be 


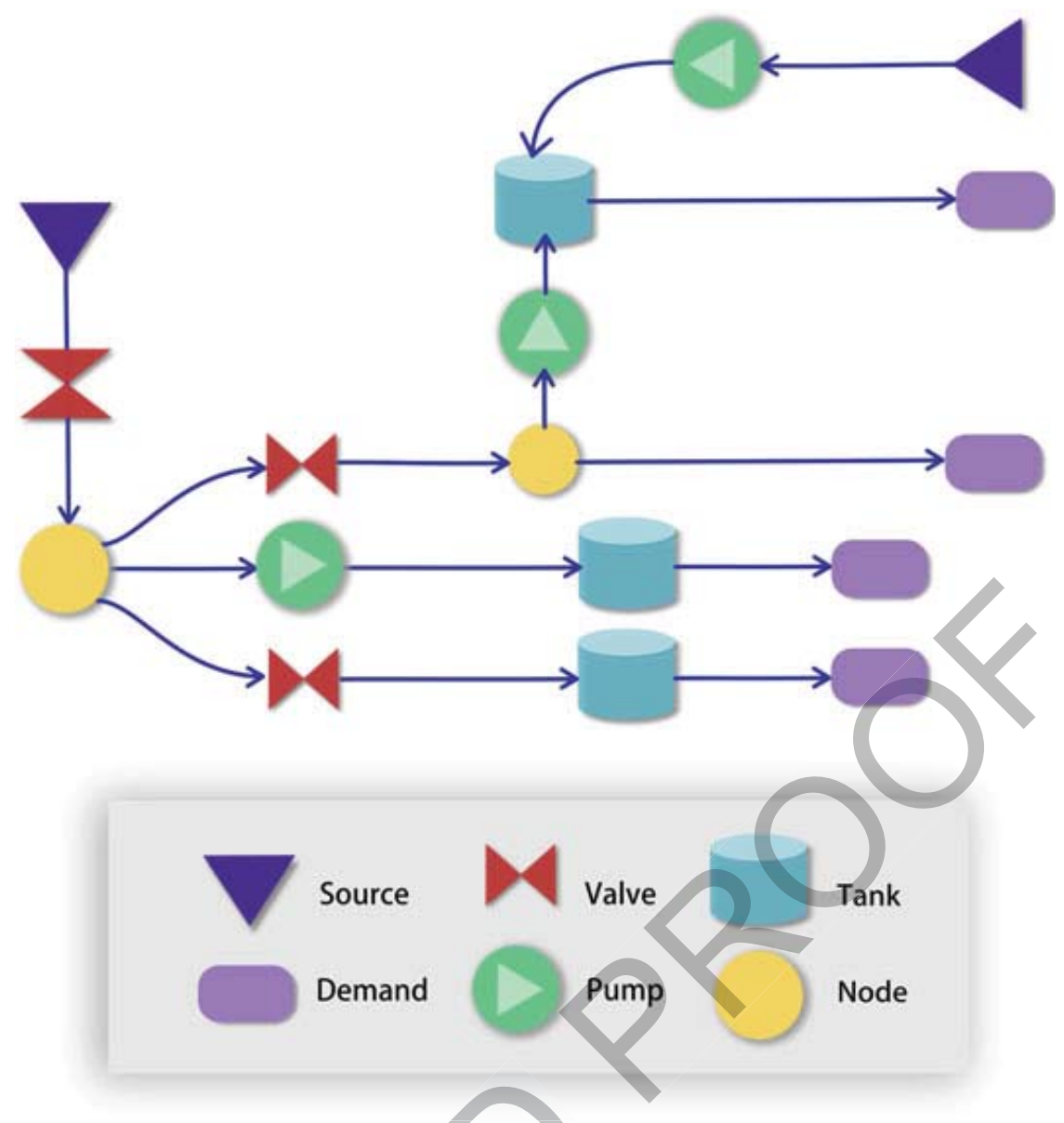

Fig. 12.2 Example of a basic topology of a generic drinking-water transport network. Note that the interaction of the main constitutive elements is shown here: sources' supply water to the system by means of pumps or valves, depending of the nature of the particular source (superficial or underground). Water is moved by using manipulated actuators in order to fill detention tanks and/or supply water to demand sectors

written as the discrete-time difference equation

$$
v_{n}(k+1)=v_{n}(k)+\Delta t\left(\sum_{j} q_{\text {in }}^{j n}(k)-\sum_{h} q_{\mathrm{out}}^{n h}(k)\right),
$$

where $q_{\text {in }}^{j n}(k)$ denotes the manipulated inflows from the $j$ th element to the $n$th tank, and $q_{\text {out }}^{n h}(k)$ denotes the manipulated outflows from the $n$th tank to the $h$ th element (which includes the demand flows as outflows). Moreover, $\Delta t$ corresponds with the sampling time and $k$ the discrete-time instant. The physical constraint related to the range of admissible storage volume in the $n$th tank is expressed as

$$
v_{n}^{\min } \leq v_{n}(k) \leq v_{n}^{\max }, \quad \text { for all } k,
$$


where $v_{n}^{\min }$ and $v_{n}^{\max }$ denote the minimum and the maximum admissible storage capacities, respectively. Notice that $\underline{v}_{n}$ might correspond with an empty tank; in practice, this value can be set as nonzero in order to maintain an emergency stored volume.

For simplicity, the dynamic behaviour of these elements is described as a function of volume. However, in most cases, the measured variable is the tank water level (by using level sensors), which implies the computation of volume taking into account the tank geometry.

\subsubsection{Actuators}

Two types of control actuators are considered: valves and pumps, or more precisely, complex pumping stations. A pumping station generally contains a number of individual pumps with fixed or variable speed. In practice, it is assumed that the flow through a pumping station is a continuous variable in a range of feasible values. The manipulated flows through the actuators represent the manipulated variables, denoted as $q_{u}$. Both pumping stations and valves have lower and upper physical limits, which are taken into account as system constraints. As in (12.2), they are expressed as

$$
q_{u_{m}}^{\min } \leq q_{u_{m}}(k) \leq q_{u_{m}}^{\max }, \text { for all } k,
$$

where $q_{u_{m}}^{\min }$ and $q_{u_{m}}^{\max }$ denote the minimum and the maximum flow capacity of the $m$ th actuator, respectively. Since this modelling is stated within a supervisory control framework, it is assumed that a local controller is available, which ensures that the required flow through the actuator is obtained.

\subsubsection{Nodes}

These elements correspond to the network points where water flows are merged or split. Thus, nodes represent mass balance relations, modelled as equality constraints related to inflows - from other tanks through valves or pumps-and outflows, the latter being not only manipulated flows but also demand flows. The expression of the mass balance in these elements can be written as

$$
\sum_{j} q_{j r}^{\text {in }}(k)=\sum_{h} q_{r h}^{\text {out }}(k),
$$

where $q_{j r}^{\text {in }}(k)$ denotes inflows from the $j$ th element to the $r$ th node, and $q_{r h}^{\text {out }}(k)$ denotes outflows from the $r$ th node to the $h$ th element. From now on, node inflows and outflows will be denoted by $q^{\text {in }}$ and $q^{\text {out }}$, even if they are manipulated variables (denoted by $q_{u}$ ). 


\subsubsection{Demand Sectors}

A demand sector represents the water demand of the network users of a certain physical area. It is considered as a measured disturbance of the system at a given time instant. The demand can be anticipated by forecasting algorithms, which are integrated within the MPC closed-loop architecture. For the case studies in this chapter, the algorithm proposed in [16], among others discussed in Chap.6, is considered. This algorithm typically uses a two-level scheme composed of

(i) a time series model to represent the daily aggregate flow values and

(ii) a set of different daily flow demand patterns according to the day type to cater for different consumption during the weekend and holiday periods. Every pattern consists of 24 hourly values for each daily pattern.

The algorithm runs in parallel with the MPC algorithm. The daily series of hourly flow predictions are computed as a product of the daily aggregate flow value and the appropriate hourly demand pattern. Regarding the daily demand forecast, its corresponding flow model is built on the basis of an ARIMA time series modelling approach described in [17]. Then, the structure of the daily flow model for each demand sensor may be written as

$$
\begin{gathered}
y_{p}(k)=-b_{1} y(k-1)-b_{2} y(k-2)-b_{3} y(k-3)-b_{4} y(k-4) \\
-b_{5} y(k-5)-b_{6} y(k-6)-b_{7} y(k-7),
\end{gathered}
$$

where the parameters $b_{1}, \ldots, b_{7}$ are estimated based on historical data. The 1-h flow model is based on distributing the daily flow prediction provided by the time series model in (12.5) using an hourly flow pattern that takes into account the daily/monthly variation as follows:

$$
y_{p h}(k+i)=\frac{y_{\text {pat }}(k, i)}{\sum_{j=1}^{24} y_{\text {pat }}(k, j)} y_{p}(k), \quad i=1, \ldots, 24,
$$

where $y_{p}(k)$ is the predicted flow for the current day $k$ using (12.5), and $y_{\text {pat }}(k)$ is the prediction provided considering the flow pattern class corresponding to the current day. Demand patterns are obtained from statistical analysis.

\subsubsection{Control-Oriented Model}

Considering the set of compositional elements described above, the control-oriented model can be obtained by joining those elements and their corresponding dynamic descriptions. In a general form, the expression which collects all these dynamics can be written as the mapping 


$$
\mathbf{x}(k+1)=\mathbf{g}(\mathbf{x}(k), \mathbf{u}(k), \mathbf{d}(k)),
$$

where $\mathbf{x} \in \mathbb{X} \subseteq \mathbb{R}^{n_{x}}$ corresponds to the system states, $\mathbf{u} \in \mathbb{U} \subseteq \mathbb{R}^{n_{u}}$ denotes the system inputs (manipulated variables) and $\mathbf{d} \in \mathbb{D} \subseteq \mathbb{R}^{n_{d}}$ denotes the system disturbances. $\mathbf{g}: \mathbb{R}^{n_{x}} \times \mathbb{R}^{n_{u}} \times \mathbb{R}^{n_{d}} \rightarrow \mathbb{R}^{n_{x}}$ is an arbitrary system state function and $k \in \mathbb{Z}_{+}$.

In the case of DWN, (12.7) is associated with the set of tank expressions in (12.1). Hence, a control-oriented discrete-time state-space model can be written as [15]

$$
\mathbf{x}(k+1)=\mathbf{A} \mathbf{x}(k)+\mathbf{B} \mathbf{u}(k)+\mathbf{B}_{p} \mathbf{d}(k)
$$

where, in particular, $\mathbf{x}$ corresponds to the water volumes $v$ of the $n_{x}$ tanks, $\mathbf{u}$ represents the manipulated flows $q_{u}$ through the $n_{u}$ actuators (pumps and valves) and d corresponds with the vector of $n_{d}$ water demands (measured disturbances affecting the system). $\mathbf{A}, \mathbf{B}$ and $\mathbf{B}_{p}$ are the system matrices of suitable dimensions. Note that since the system control-oriented model of a DWN does not collect the static dynamics described by DWN nodes in (12.4), then (12.8) can be further rewritten as

$$
\begin{aligned}
\mathbf{x}(k+1) & =\mathbf{A} \mathbf{x}(k)+\boldsymbol{\Gamma} \boldsymbol{\mu}(k), \\
{\left[\mathbf{E}_{u} \mathbf{E}_{d}\right] \boldsymbol{\mu}(k) } & =0
\end{aligned}
$$

where $\boldsymbol{\Gamma}=\left[\begin{array}{ll}\mathbf{B} & \mathbf{B}_{p}\end{array}\right], \boldsymbol{\mu}(k)=\left[\begin{array}{ll}\mathbf{u}(k)^{T} & \mathbf{d}(k)^{T}\end{array}\right]^{T}$ and $\mathbf{E}_{u}, \mathbf{E}_{d}$ are matrices of suitable dimensions. It can be seen that (12.9a) comes from the mass balance in tanks, while (12.9b) comes from the network nodes. Also notice that when all the network flows are manipulated, then $\mathbf{A}$ is an identity matrix of suitable dimensions.

\subsubsection{Control Criteria}

It is possible to use different control objectives depending on the operational goals considered by the network managers. This section describes the most common control objectives and the resultant multi-objective cost function. Therefore, this chapter considers and discussed the following control objectives [15, 18].

\subsubsection{Minimization of Water Production and Transport Costs}

The main economic costs associated with drinking-water production are due to treatment processes, water acquisition or use costs and, most importantly, to electricity costs associated with pumping. Delivering this drinking water to appropriate pressure levels through the network involves important electricity costs in booster pumping as well as elevation from underground devices. In a specific case, this objective can be mathematically formulated as the minimization of 


$$
J_{1}(k) \triangleq\left(\boldsymbol{\alpha}_{1}+\boldsymbol{\alpha}_{2}(k)\right)^{T} \mathbf{u}(k)
$$

where $\boldsymbol{\alpha}_{1}$ corresponds to a known vector related to water production costs, depending on the selected water source, and $\boldsymbol{\alpha}_{2}(k)$ is a vector of suitable dimensions associated with the energy pumping costs. Note the $k$-dependence of $\boldsymbol{\alpha}_{2}$ since the pumping cost has different values according to the variable electric tariffs along a day.

\subsubsection{Appropriate Management of Safety Water Storage}

The satisfaction of water demands must be fulfilled at all times. However, some risk prevention mechanisms need to be introduced in the tank management so that, additionally, the stored volume is preferably maintained above certain safety value for eventual emergency needs and to guarantee future water availability. Therefore, this objective may be achieved by minimizing the following expression:

$$
J_{2}(k)= \begin{cases}\left(\mathbf{x}(k)-\mathbf{x}^{\mathrm{safe}}\right)^{T}\left(\mathbf{x}(k)-\mathbf{x}^{\mathrm{safe}}\right) & \text { if } \mathbf{x}(k) \leq \mathbf{x}^{\mathrm{safe}} \\ 0 & \text { otherwise }\end{cases}
$$

where $\mathbf{x}^{\text {safe }}$ is a term which determines the safety volume to be considered for the control law computation. This term might appear as unnecessary given the guarantees of the MPC design but since a trade-off between the other costs and the volumes is present, the controller would tend to keep the lowest possible the tank water volumes. This fact would reduce the safety of the system to handle unexpected extra demands, such as fire extinction, among others.

\subsubsection{Smoothing of Control Actions}

Valves must also operate smoothly in order to avoid big transients in the pressurized pipes. This fact could lead to poor pipe condition. The use of a smooth reference changes also helps the lower-level regulator performance. Similarly, water flows requested from treatment plants must have a smooth profile due to plants operational constraints. To obtain such smoothing effect, control signal variation between consecutive time intervals is therefore penalized. The penalty term to be minimized is

$$
J_{3}(k)=\Delta \mathbf{u}(k)^{T} \Delta \mathbf{u}(k),
$$

where $\Delta \mathbf{u}(k) \triangleq \mathbf{u}(k)-\mathbf{u}(k-1)$.

\subsubsection{Multi-objective Performance Function}

The multi-objective performance function $\mathcal{J}(k)$ that gathers the aforementioned control objectives, either in the case of DWN or SN, can be written as 


$$
\mathcal{J}(k)=\sum_{j=1}^{\varphi} \gamma_{j} J_{j}(k)
$$

where a set of $\varphi$ control objectives are considered, and, in turn, a multi-objective open-loop optimization problem (OOP) is stated. The prioritization of the control objectives is performed by using the order of the mathematical cost function associated with each objective and also a set of appropriate weights $\gamma_{j}$. These weights are selected offline by means of trial-and-error procedures, taking into account the priority of each objective within the cost function. More sophisticated tuning methodologies for tuning multi-objective control problems based on lexicographic minimizers [19], goal programming [20] or Pareto-front computations [21] may be also considered.

\subsubsection{MPC Problem Formulation}

Collecting the parts described in previous subsections, the MPC design follows the traditional procedures presented in $[11,12,22]$, which involve solving an optimization problem over a prediction horizon $H_{p}$, where a cost function is minimized subject to a set of physical and operational constraints. Once the minimization is performed, a vector of $H_{u}$ control actions over $H_{p}$ is obtained. Only the first component of that vector is considered and applied to the plant. The procedure is repeated for the next time instant taking into account the feedback measurements coming from the system, following the classic receding horizon strategy.

In general terms, the MPC controller design is based on the solution of a OOP

$$
\mathcal{V}\left(k, H_{p}\right)=\min \sum_{i=0}^{H_{p}} \sum_{j=1}^{\varphi} \gamma_{j} J_{j}(k+i \mid k),
$$

subject to the system model and the physical and operational constraints, where $H_{p}$ corresponds to the prediction horizon, and index $k$ represents the current time instant, while index $i$ represents the time instant along $H_{p}$. Hence, notation $k+i \mid k$ denotes the time instant $k+i$ given $k$. Note that (12.14) corresponds with (12.13) over the prediction horizon.

According to the case, the minimum of $\mathcal{V}\left(k, H_{p}\right)$ is achieved by finding a set of optimal variables which generally correspond with the manipulated variables of the system model but that could include further variables of diverse nature. Hence, for a prediction window of length $H_{p}$ and considering $\mathbf{z} \in \mathbb{R}^{s H_{p}}$ as the set of $s$ optimization variables for each time instant over $H_{p}$, the multi-objective optimization problem can be formulated as 


$$
\begin{gathered}
\min _{\left\{\mathbf{z} \in \mathbb{R}^{s H_{p}}\right\}} f(\mathbf{z}) \\
\text { subject to } \\
H_{1}(\mathbf{z}) \leq 0, \\
H_{2}(\mathbf{z})=0,
\end{gathered}
$$

where $f(\mathbf{z})$ comes from the manipulation of (12.14). Moreover, $H_{1}(\mathbf{z})$ and $H_{2}(\mathbf{z})$ are vectors of dimensions $r_{i} H_{p} \times 1$ and $r_{e} H_{p} \times 1$, respectively, containing the constraint functions. Here, $r_{i}$ is the number of inequality constraints, and $r_{e}$ is the number of the problem equality constraints. It can be observed that (12.15b) and (12.15c) gather all problem constraints including those from the system model, the physical restrictions of its variables and the operational and management constraints.

Assuming that the OOP (12.15) is feasible for $\mathbf{z} \in \mathbb{R}^{s H_{p}}$, there exists an optimal solution given by the sequence

$$
\mathbf{z}^{*} \triangleq\left(\mathbf{z}^{*}(0 \mid k), \mathbf{z}^{*}(1 \mid k), \ldots, \mathbf{z}^{*}\left(H_{p} \mid k\right)\right)
$$

and then the receding horizon philosophy sets [12]

$$
\mathbf{z}_{\mathrm{MPC}}(\mathbf{x}(k)) \triangleq \mathbf{z}^{*}(0 \mid k)
$$

and disregards the computed inputs from $k=1$ to $k=H_{p}$, repeating the whole process at the following time step. Equation (12.17) is known as the MPC law.

Therefore, the MPC problem formulation in DWNs gives the expressions for each of the problem parts described above. Thus, mapping (12.7) must be replaced by the system modelling in (12.9) when treating a DWN. Finally, constraints in (12.15b) and (12.15c) are conveniently expressed taking into account the type of network and its constitutive components; for example, constraints in (12.9b) must be included when a DWN is considered. Constraints (12.2) and (12.3) are always included. In order to manage the uncertainty of the system disturbances over the prediction horizon, a suitable approach is the stochastic paradigm, which includes explicit models of uncertainty/disturbances in the design of control laws and by transforming hard constraints into probabilistic constraints. As reviewed in [23], the stochastic approach is a classic one in the field of optimization, and a renewed attention has been given to the stochastic programming [24], as a powerful tool for robust control design, leading to the stochastic MPC and specially to the chanceconstrained MPC (CC-MPC) [25] (see Chap. 13).

\subsection{Simulations and Results}

As an application case study to show the performance of the proposed modelling and control approach, some results of its application offline (in simulation) in several real scenarios in the Barcelona WTN are presented. A simulator of this network has 
been built using MATLAB/Simulink and validated using real data coming from real scenarios (see Figs. 12.10 and 12.11 and the corresponding explanations in Chap. 2). This allows testing the controller against a virtual reality introducing, for example, real demand in the simulator different from the predicted demand used by the controller. The MPC controller was implemented with the PLIO tool presented in [26] that uses GAMS/CONPOPT solver to solve the corresponding optimization problem. This general-purpose decision support tool has been developed to allow the user to implement optimal/predictive control techniques in large-scale drinking-water systems (see Fig. 12.3).

The modelling and predictive control problem solution algorithms are designed for real-time decision support, in connection with a SCADA system. The hydraulic modelling relies on simple, but representative enough, dynamic equations whose parameters are recalibrated online using recursive parameter estimation and real data obtained from sensors in the network. Demand forecast models, based on time series analysis, are also dynamically updated. The real-time calibration using recursive parameter estimation methods contributes to deal with hydraulic uncertainty. This modelling choice, as well as the optimization method selection, allows to deal with very large-scale systems. Another distinguishing feature is its capability to accommodate complex operational goals.

In Fig. 12.4, the evolution of volume at a number of tanks is shown. The simulator output is shown in blue, while red is used for the real data. In some cases, small discrepancies between both volume curves are not associated with modelling errors but with errors in real data due to a faulty sensor. The most important conclusion after

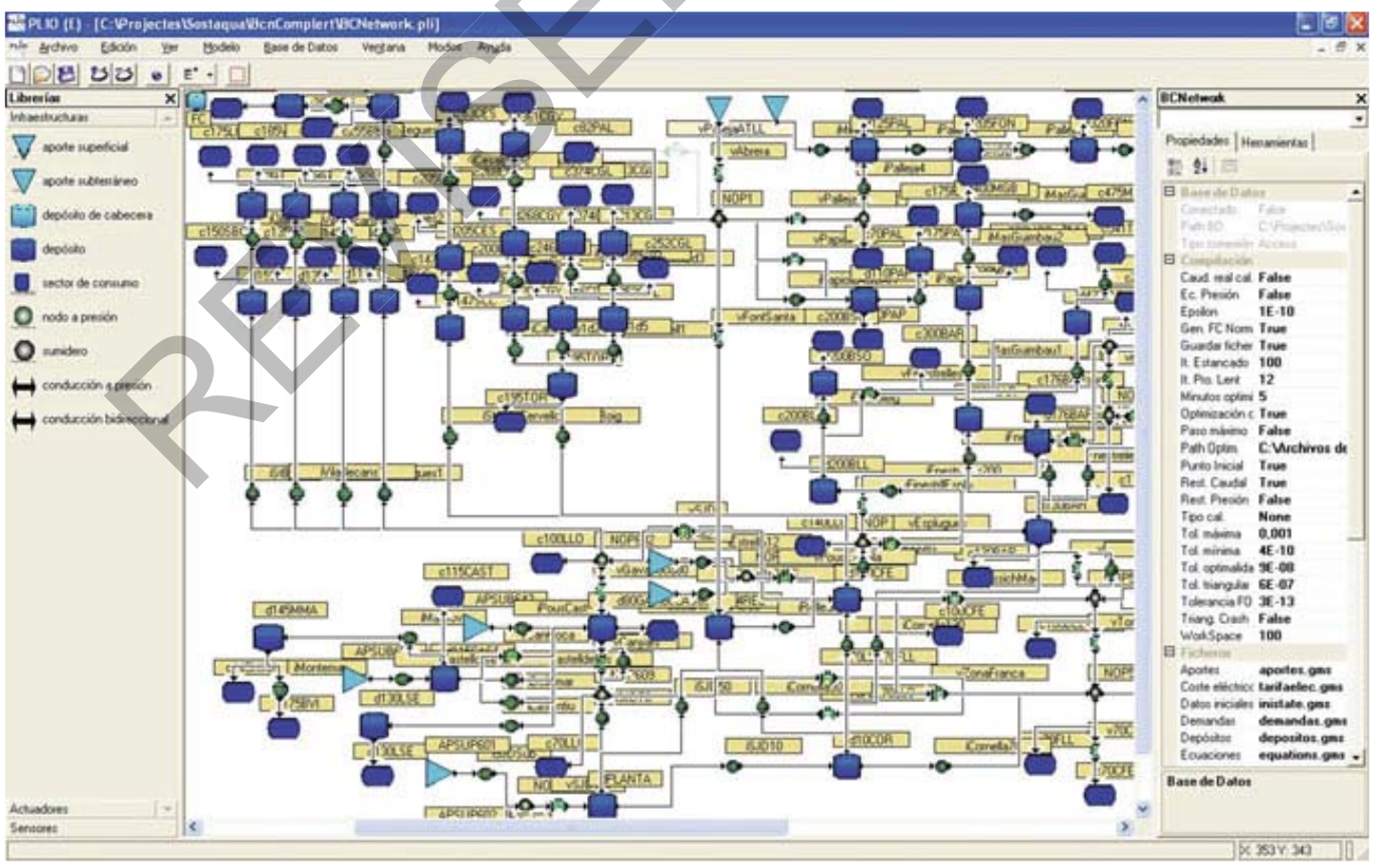

Fig. 12.3 PLIO interface corresponding to the model manager module allows creating/updating the model of the water network in a user friendly way 

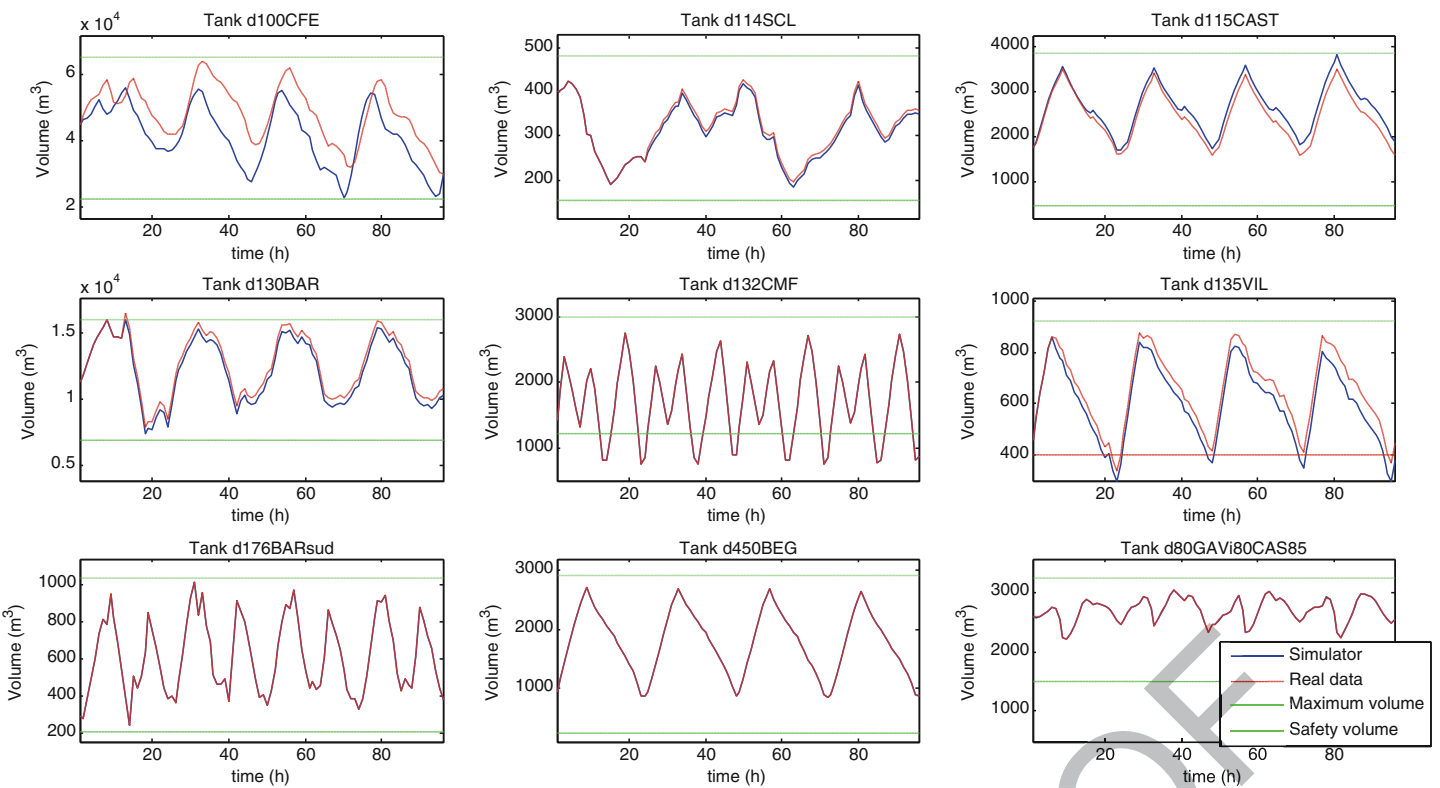

Fig. 12.4 Model validation based on the comparison between real yolumes and the simulated ones

this process is that this simulator allows making the model validation process easier. The model has been validated and accepted by Aguas de Barcelona as representative of the network real behaviour.

The Barcelona WTN is organized in different pressure levels. Figure 12.5 presents the several pressure levels in different colours. Each sector will be supplied through a

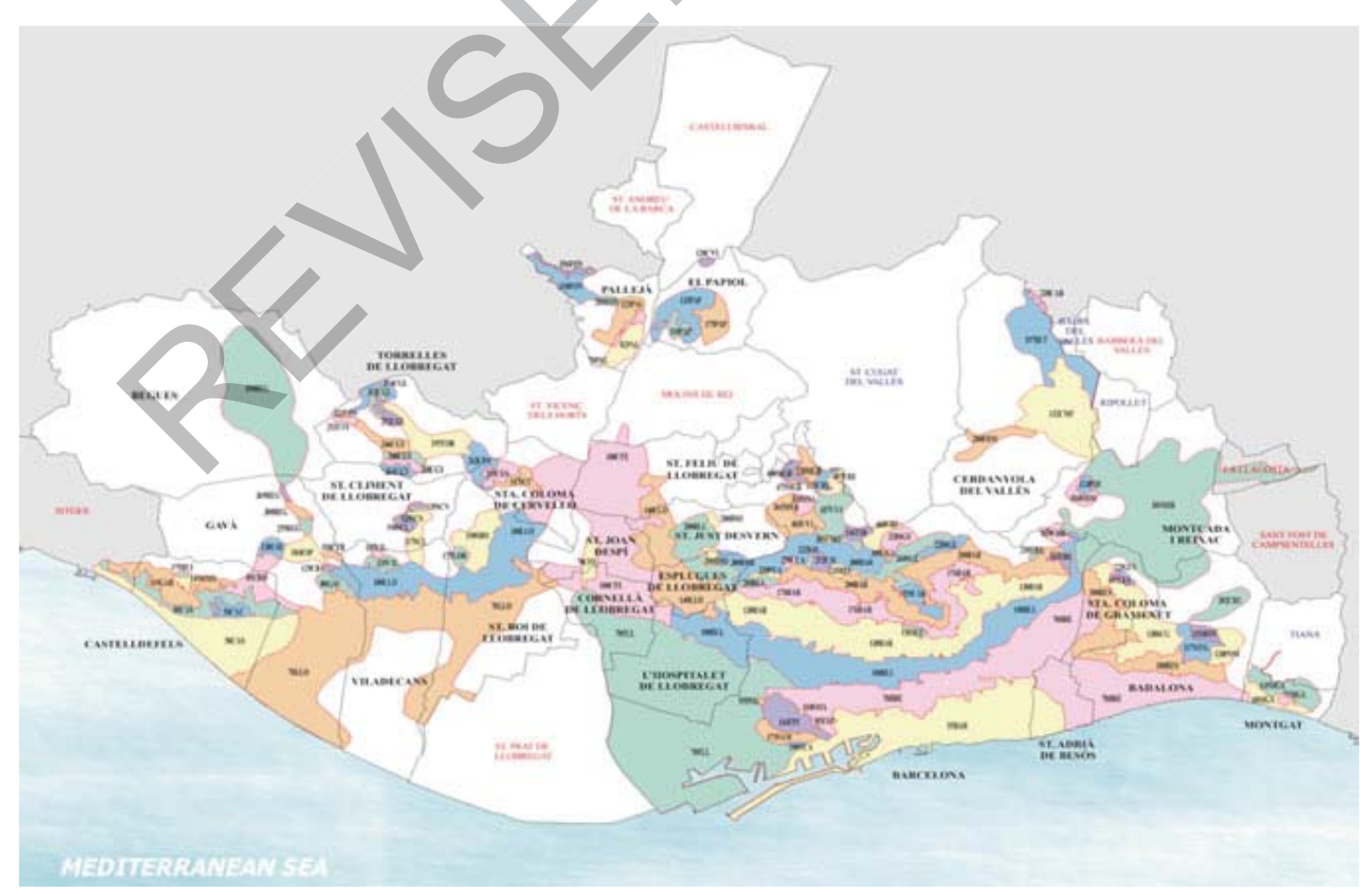

Fig. 12.5 Barcelona water network demand sectors 

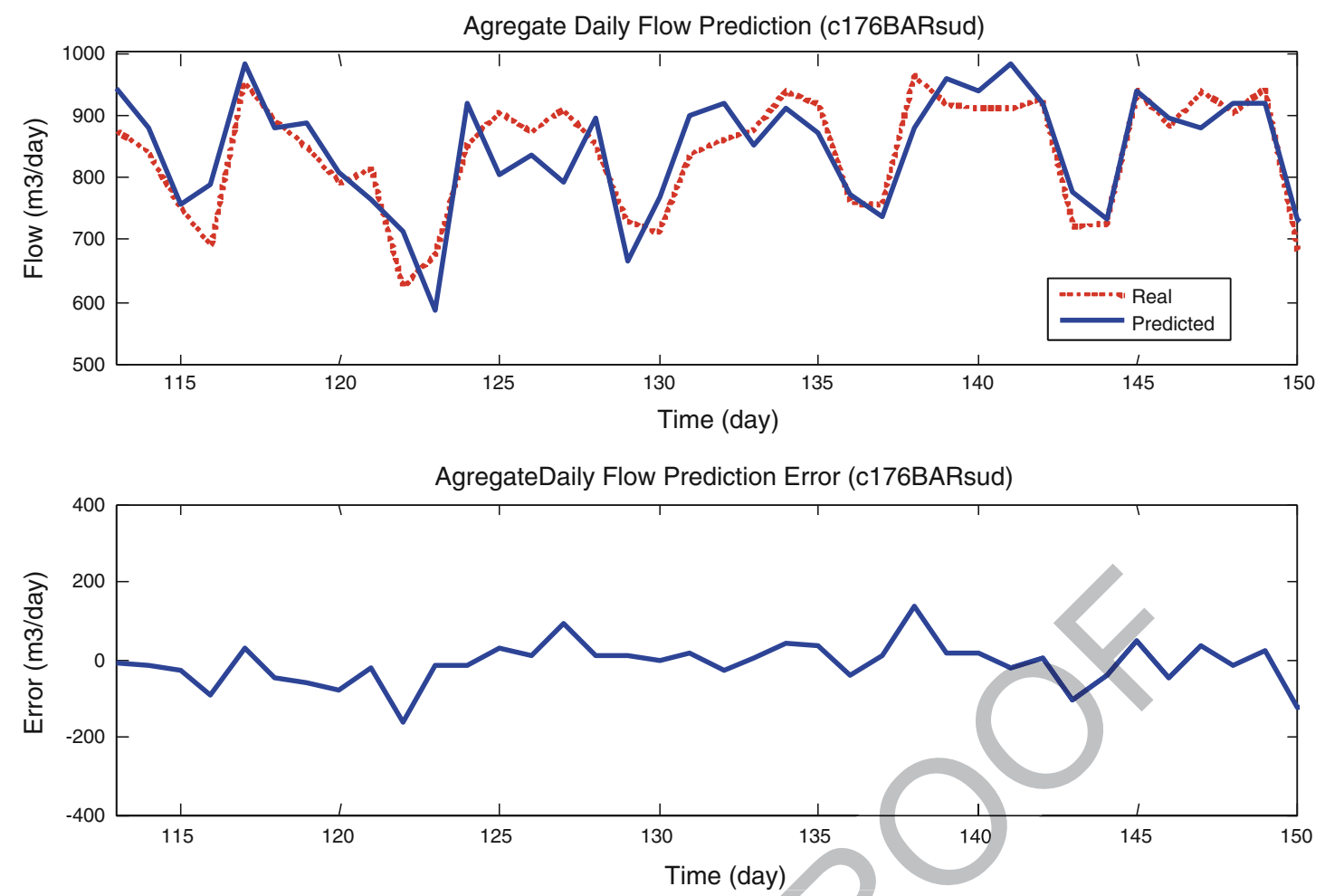

Fig. 12.6 Validation of the aggregate daily demand forecast corresponding to the sector c176BARsud

storage tank. The distribution network that connects each storage tank with individual consumers will not be modelled in detail but will be summarized as an aggregated demand. Each demand will be modelled using a time series pattern. Figures 12.6 and 12.7 show the validation of the daily and hourly demand forecast in the sector c176BARsud using the demand forecast algorithm presented in Sect. 12.3.1.4.

\subsubsection{Test Scenarios}

To test and adjust the MPC controller, different scenarios have been chosen. The main difference between the selected scenarios is related to source operation. So, the objectives of this study are as follows:

- to compare the effects of the MPC strategies with those of the currently applied control strategies and

- to show the effects of source management in the total operation cost, including electrical and water costs.

With reference to source management, two different scenarios are shown:

- Scenario 1: Scheduled flow. In this case, the flow of all sources is fixed to real values obtained from real historical data. 

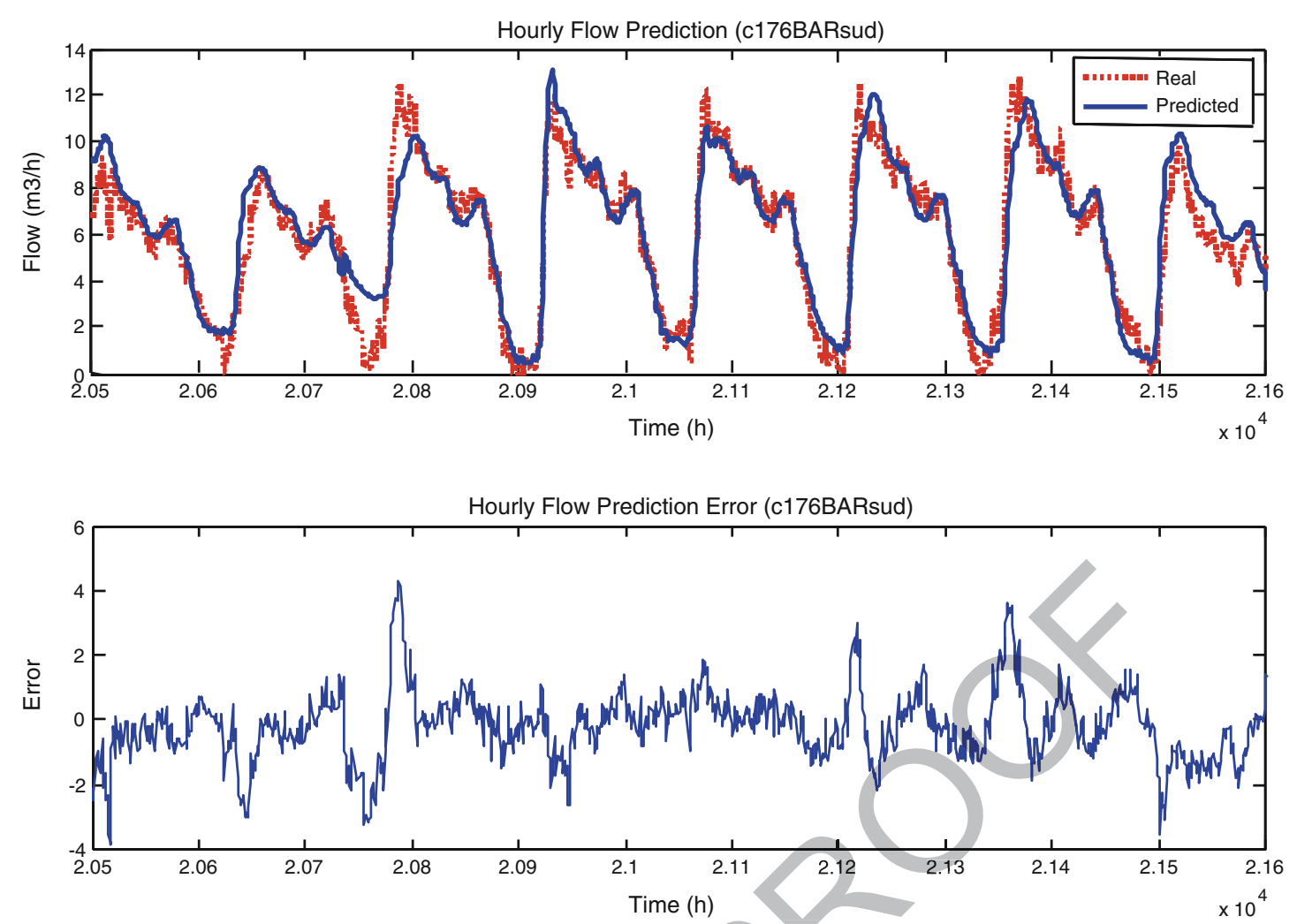

Fig. 12.7 Validation of the hourly demand forecast corresponding to the sector c176BARsud

- Scenario 2: Flow optimization. The optimizer calculates the flow to be abducted from each source at each time step, taking into account its operational limits, according to long-term planning.

- Scenario 3: Fixing main source. The main source of water is fixed, while the others are optimized.

The parameters taken into account for the calibration of the model are the initial volumes and safety storage volumes in tanks, as well as the objective function weights for each of the operational goals (the economical, safety and smoothness factors). Objective function weights are calibrated by experimentally analysing their effects on the compromise between the operational goals, with historic data. In [21], the authors have explored multi-objective optimization techniques to tune them in a more sophisticated way. Tank initial and safety storage volumes are taken from real historic data of each scenario, in order to make optimization results comparable with current control strategy.

The period in both scenarios is $96 \mathrm{~h}$ (4 days), and all of them correspond to the same period, between July 23 and July 26 of 2007. It means that the demand is the same in both scenarios, so they are comparable. To estimate the demand of each sector, the demand forecast method presented in Sect.12.3.1.4 is used. The total demanded volume for each day is obtained from the total contribution from each source. In Table 12.1, values of volume per day are shown. 
Table 12.1 Total input volume for studied days

\begin{tabular}{l|l|l}
\hline Date & Total input volume $\left(\mathrm{m}^{3}\right)$ & Mean flow $\left(\mathrm{m}^{3} / \mathrm{s}\right)$ \\
\hline $23 / 07 / 2007$ & 633694 & 7.334 \\
\hline $24 / 07 / 2007$ & 668136 & 7.733 \\
\hline $25 / 07 / 2007$ & 617744 & 7.150 \\
\hline $26 / 07 / 2007$ & 627406 & 7.262 \\
\hline & Mean & 7.370 \\
\hline
\end{tabular}

\subsubsection{Results and Discussion}

In all the test scenarios, the MPC controller computed solutions to meet demands and operational constraints at all times, while optimizing the operational goals. Some illustrative results of the MPC application on the complete Barcelona WTN are presented in this section. For these tests, the same model is used.

\subsubsection{Scenario 1: Scheduled Flow}

In this first scenario, source flows are imposed using real data obtained from Aguas de Barcelona historical database. The interesting point of this scenario is the comparison between MPC control strategy and current control strategy: water sources management is the same in both cases. This scenario is used to show the potential of MPC for minimizing the electrical (pumping) cost. The evolution of source flows is shown in Fig. 12.8.

In Table 12.2, electrical and water cost in percentage of the total cost for the current control strategy are shown. In Table 12.3, costs for the MPC control as an increase or decrease percentage with regard to current control are presented.

Water production cost (acquisition and treatment) represents a value near $70 \%$ of the total cost, and there is no variation of this cost in the MPC control because of the fixed sources. With regard to electrical cost, the improvement is between 10 and $25 \%$, which represents a decrease of the total cost between 3 and $8 \%$. To show the differences between the current control and the MPC control, some tank volume and

Table 12.2 Current control strategy costs in percentage

\begin{tabular}{l|l|l|l}
\hline Date & Electricity cost & Water cost & Total cost \\
\hline $23 / 07 / 2007$ & 33.13 & 66.87 & 100.00 \\
\hline $24 / 07 / 2007$ & 34.66 & 65.34 & 100.00 \\
\hline $25 / 07 / 2007$ & 32.00 & 68.00 & 100.00 \\
\hline $26 / 07 / 2007$ & 31.29 & 68.71 & 100.00 \\
\hline
\end{tabular}



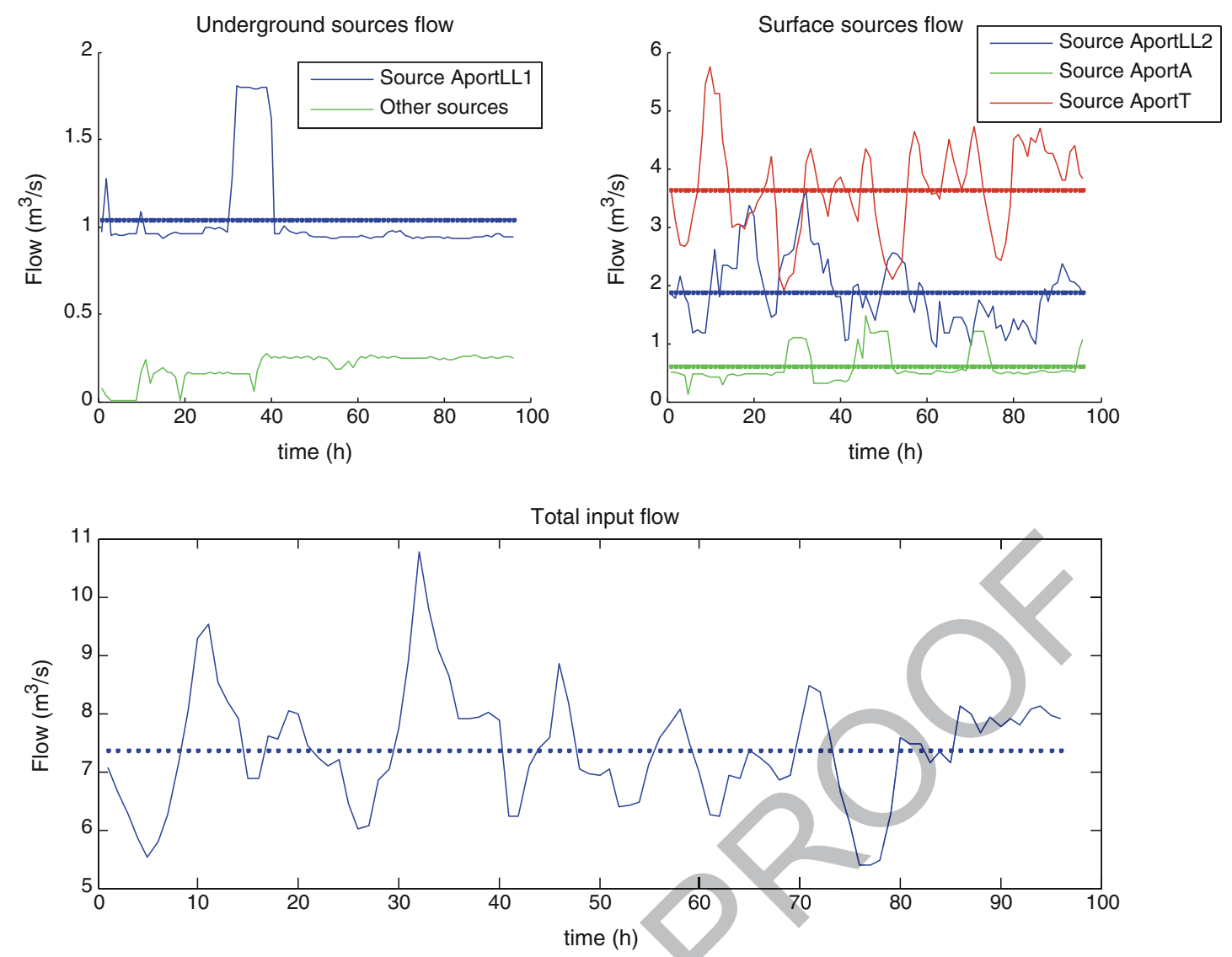

Fig. 12.8 Sources' flow evolution for Scenario 1: scheduled flow

Table 12.3 MPC improvement in percentage for Scenario 1 (scheduled flow) regarding Table 12.2 values

\begin{tabular}{l|l|l|l}
\hline Date & Electricity cost & Water cost & Total cost \\
\hline $23 / 07 / 2007$ & -23.27 & +0.00 & -7.71 \\
\hline $24 / 07 / 2007$ & -10.56 & +0.00 & -3.66 \\
\hline $25 / 07 / 2007$ & -20.61 & +0.00 & -6.59 \\
\hline $26 / 07 / 2007$ & -18.58 & +0.00 & -5.81 \\
\hline
\end{tabular}

actuator flow plots are shown. In Fig. 12.9, some tank volume evolution can be seen, as well as maximum and security volumes.

The smoothness term is not the only factor with effects on pumps' operation. The electric tariff for each pump is another factor that affects pump operation in order to minimize electrical cost. In Fig. 12.10, the effects of the electricity cost are shown. It can be seen that if it is possible, pumps only run during the cheapest period (e.g., iPalleja1). In cases where with a maximum flow during off-peak hours the necessary volume is not reached, pumps must work during other periods. Pump iFnestrelles200 is an example of this case. Since it is not enough to pump during the cheapest period, this pump is pumping during the medium-cost period too, but with a maximum flow lower than in the cheapest one. 

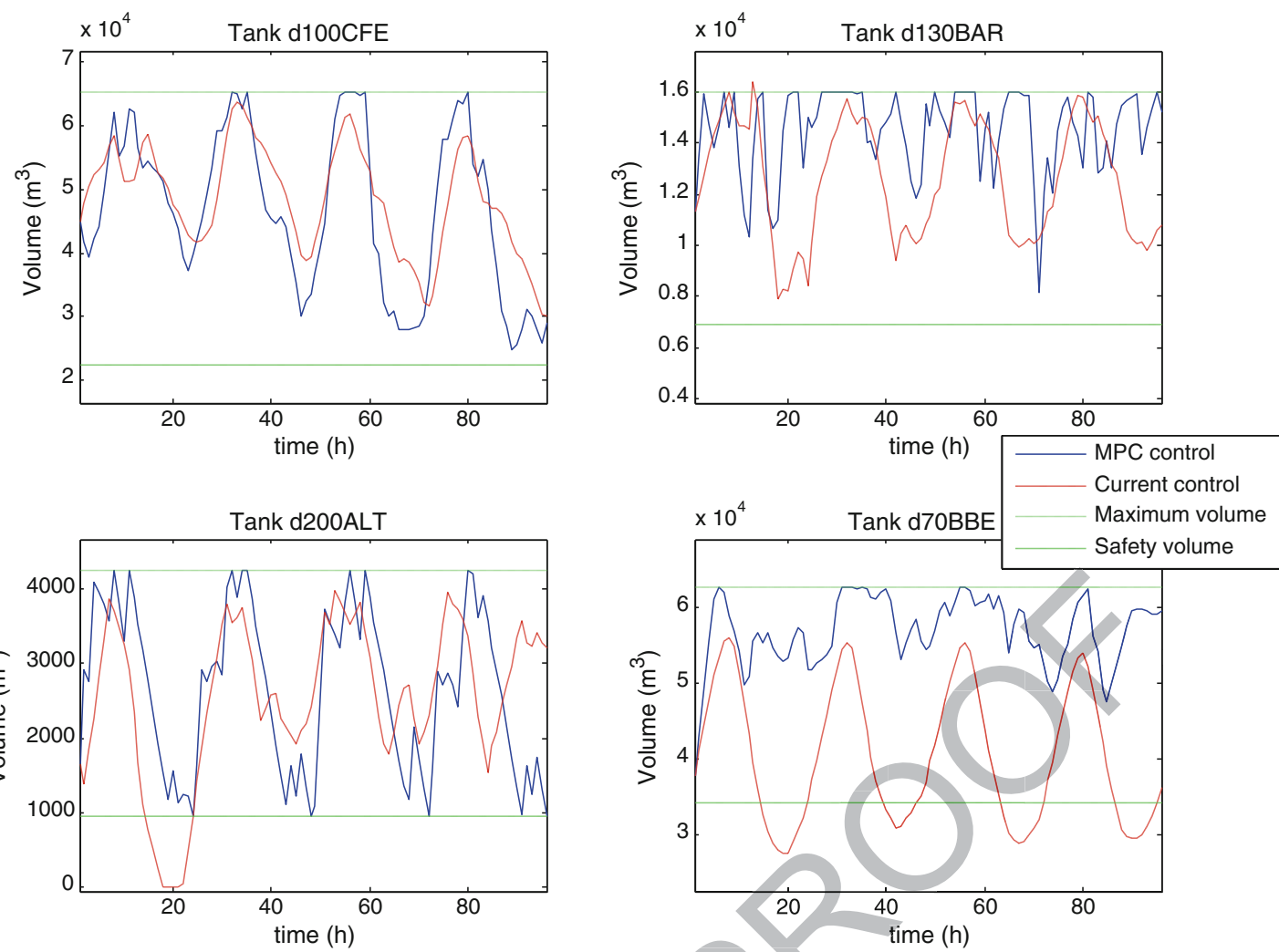

Fig. 12.9 Some tanks' volume evolution: current control and MPC control comparison

\subsubsection{Scenario 2: Flow Optimization}

In this second scenario, the source flows are optimized. It means that the only limitation is the minimum and the maximum flow of actuators in the output of each source. In this case, both electrical and water cost are optimized, so it is expected to obtain a higher improvement in the total cost referring to the Scenario 1, where sources' flow was fixed. This scenario represents a theoretical solution of the water management in the Barcelona WTN. Indeed, the optimization carried out gives total freedom to the different sources, while on a real situation, sources are not unlimited or unrestricted: its availability as well as its future guarantee compromises the total amount of water entering the system from each source. Therefore, the hereby shown results give us an idea of how far flow optimization could go if there were no sources' restrictions. In Fig. 12.11, sources' flow evolution is shown. As it can be seen, Llobregat's mean flow is about $5 \mathrm{~m}^{3} / \mathrm{s}$ (which is the maximum possible contribution of this source), while the lack of water necessary to satisfy the total demand is taken from Ter and Abrera. Underground sources' water cost is penalized to avoid its overexploitation.

Electrical and water cost obtained in this scenario is compared with both the current control case and the MPC case of Scenario 1 (scheduled flow). In Tables 12.4 and 12.5 , this comparison is shown.

The first point to emphasize is the high water improvement, between 30 and $50 \%$. As shown, it seems that maximizing water taken from Llobregat, water cost is clearly decreased. On the other hand, electrical cost is increased, but the decrease of 

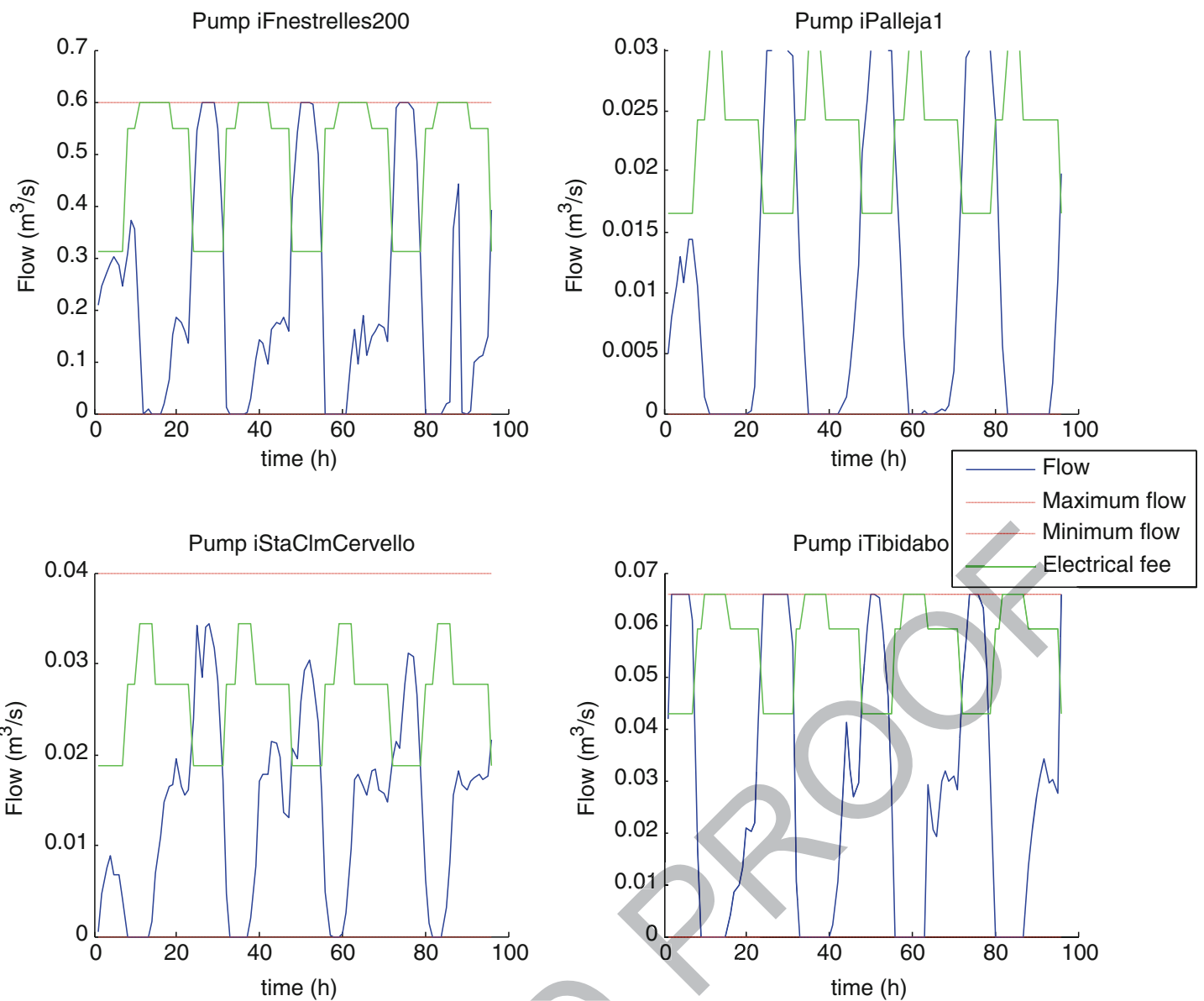

Fig. 12.10 Electrical fee effects on pumps operation

Table 12.4 Scenario 2 improvement with regard to current control case (Table 12.2)

\begin{tabular}{l|l|l|l}
\hline Date & Electricity cost & Water cost & Total cost \\
\hline $23 / 07 / 2007$ & 18.92 & -50.70 & -27.63 \\
\hline $24 / 07 / 2007$ & 14.04 & -32.56 & -16.41 \\
\hline $25 / 07 / 2007$ & 26.29 & -43.91 & -21.45 \\
\hline $26 / 07 / 2007$ & 26.09 & -44.43 & -22.36 \\
\hline
\end{tabular}

Table 12.5 Scenario 2 improvement with regard to Scenario 1 case (scheduled flow)

\begin{tabular}{l|l|l|l}
\hline Date & Electricity cost & Water cost & Total cost \\
\hline $23 / 07 / 2007$ & 54.99 & -50.70 & -21.59 \\
\hline $24 / 07 / 2007$ & 27.51 & -32.56 & -13.23 \\
\hline $25 / 07 / 2007$ & 59.08 & -43.91 & -15.91 \\
\hline $26 / 07 / 2007$ & 54.86 & -44.43 & -17.57
\end{tabular}



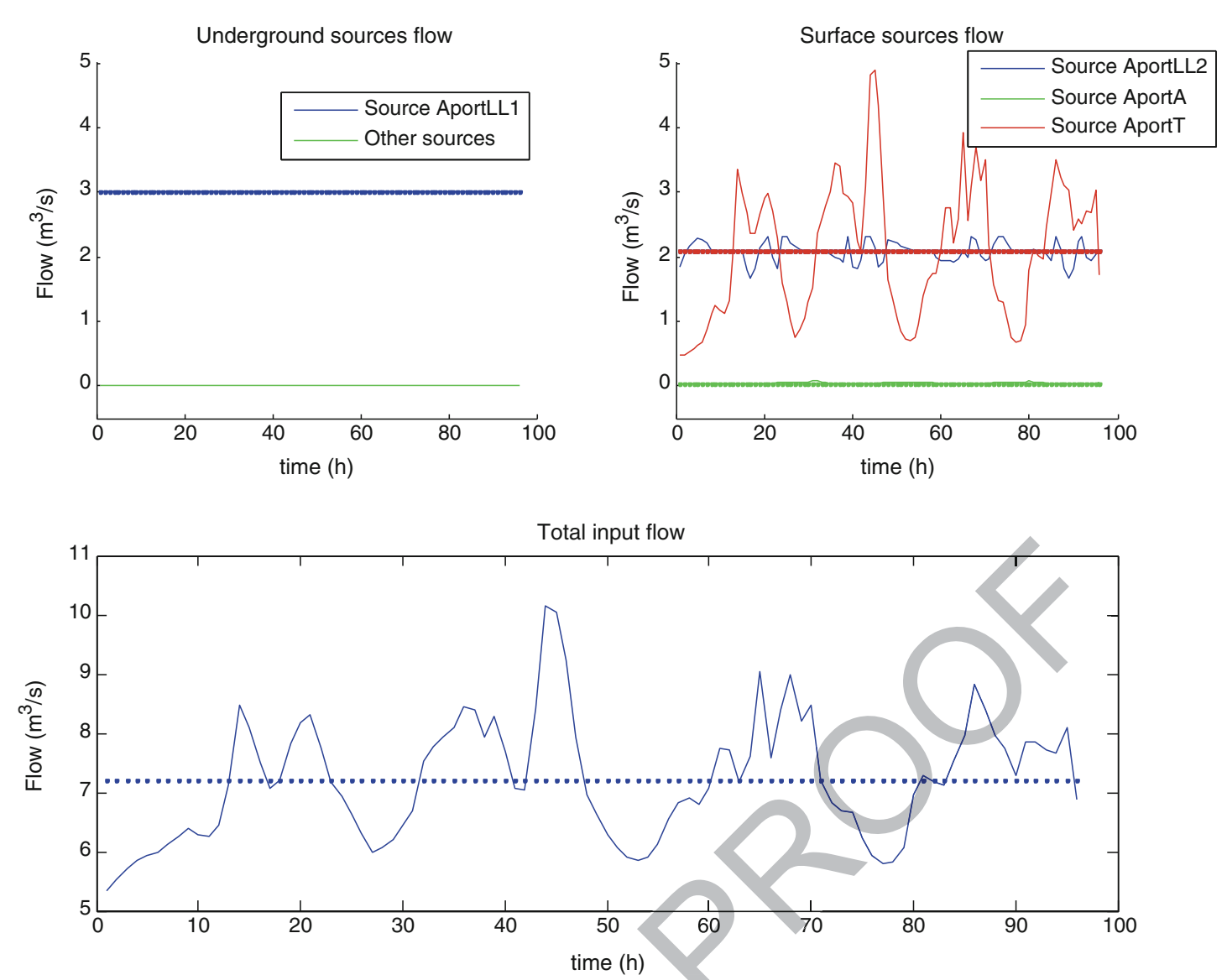

Fig. 12.11 Sources' flow evolution for Scenario 2: flow optimization

the total cost in this second scenario regarding current control case and Scenario 1 is important.

\subsubsection{Scenario 3: Fixing Main Source}

The two main sources of the Barcelona water network are the Llobregat and Ter rivers. Barcelona's average demand is about $7.5 \mathrm{~m}^{3} / \mathrm{s}$. For ecological reasons, Aguas de Barcelona company uses Llobregat source at its maximum capacity in which value depends on the river flow. The rest of flow is supplied by Ter source. From Fig. 12.12, it can be noticed that both sources affect the economic cost in an inverse way. Increasing the amount of water extracted from Llobregat source reduces the water cost while increasing the electrical cost. On the other hand, the Ter source behaves on the opposite sense: increasing the amount of water extracted from this river reduces the electrical cost while augmenting the water cost. The reason for this behaviour is due to a smaller water price in the case of Llobregat. But, since Llobregat source is located close to the sea level, while Ter source is in the upper part of the city, electrical costs will be higher in case of the Llobregat source since more pumping will be required to supply water from this source. In the case when 

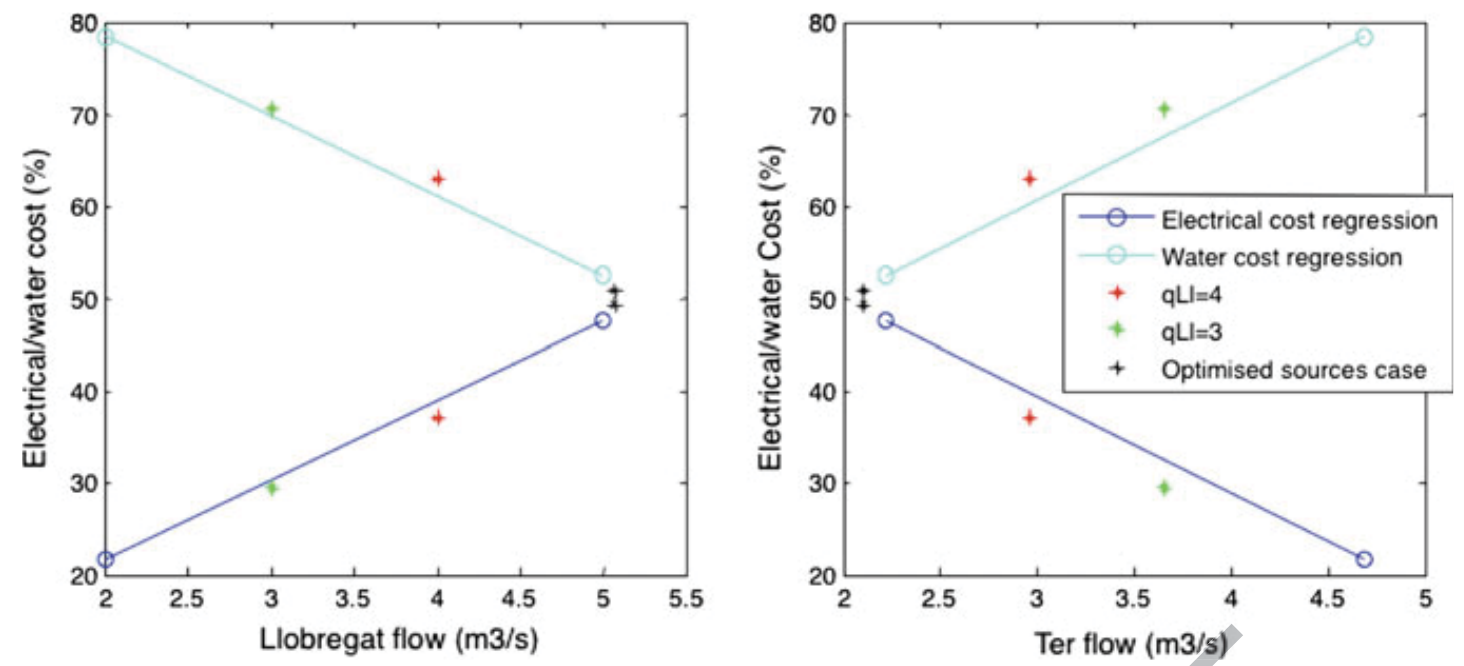

Fig. 12.12 Electrical and water cost when fixing Llobregat source

sources are not fixed, the optimal combination leads to take most of the water from Llobregat source and the remaining from the Ter source.

\subsubsection{Complementary Comments}

In Table 12.6, a brief summary of results presented is shown, as a mean value of four days of study. The costs of Scenarios 1 and 2 are referred to current control values.

From this table, conclusions that can be emphasized are as follows:

- Maximizing the flow from the source Llobregat to optimize total cost.

- Flow optimization allows higher improvement with regard to fixed real flows because the optimizer can maximize Llobregat's flow contribution if it is possible. Sometimes, it is not possible because of the reasons not related to network characteristics (operational limits of actuators and tanks).

- Ter total cost (only water cost because there is no pump) is higher than the Llobregat one (water and electrical cost associated). This fact, sources' behaviour and results of both test scenarios indicate that:

Table 12.6 Summary of results for scenarios presented

\begin{tabular}{l|l|l|l}
\hline Cost & Current control $(\%)$ & Scenario 1 $(\%)$ & Scenario 2 $(\%)$ \\
\hline Electrical & 32.77 & -18.26 & +21.34 \\
\hline Water & 67.23 & 0 & -42.90 \\
\hline Total & 100 & -5.94 & -21.96 \\
\hline
\end{tabular}


- Reduction of electrical cost involves reduction of the contribution from Llobregat.

- Reduction of water cost involves reduction of Ter source contribution.

- Total cost is minimized by maximizing Llobregat source contribution.

\subsection{Conclusions}

MPC techniques provide useful tools for generating water management strategies in large and complex water networks, which may be used for decision support, as well as for fully automated control of a water network. This work describes the use of MPC for flow management in a large water system, involving supplies, production plants and water transport into the distribution areas. The chapter presents the application of a unified approach to the water system management including supplies, production, transport and distribution areas. The modelling and predictive control solutions are designed for real-time decision support. The hydraulic modelling relies on simple, but representative, dynamic equations and recursive real-time parameter calibration using updated data from telemetry. Demand predictions are also dynamically updated. The potential of these techniques for real-time control of water supply and distribution has been shown with two representative examples of complex operational situations. The test scenarios are based on real situations which are known to have caused difficulties to operators and, in some cases, severe effects on the service to consumers. The application described in the chapter deals with these scenarios successfully, by producing control strategies that rearrange flows, production plant levels, pumping from underground sources, etc., in a way that demands are met at all times with improved results with respect to management goals. This type of decision support is extremely useful for water system operators in large-scale systems, especially those involving several different water management levels (supply, production, transport and distribution), where the control solutions may not obvious are successfully implemented.

\section{References}

1. Tu M, Tsai F, Yeh W (2005) Optimization of water distribution and water quality by hybrid genetic algorithm. J Water Resour Planning Manag 131(6):431-440

2. Nitivattananon V, Sadowski E, Quimpo R (1996) Optimization of water supply system operation. J Water Resour Planning Manage 122(5):374-384

3. Westphal KS, Vogel RM, Kirschen P, Chapra SC (2003) Decision support system for adaptive water supply management. J Water Resour Planning Manag 129(3):165-177

4. Advances in water supply management (2003) Swets and Zeitlinger, The Netherlands

5. Brdys MA, Ulanicki B (1994) Operational control of water systems: structures, algorithms and applications. Prentice Hall 
6. Cembrano G, Wells G, Quevedo J, Pérez R, Argelaguet R (2000) Optimal control of a water distribution network in a supervisory control system Control Engineering Practice, 8(10): 11771188

7. Butler D, Memon F (2006) Water demand management. IWA Publishing, London

8. Marinaki M, Papageorgiou M (2005) Optimal real-time control of sewer networks. Springer, Secaucus, NJ (USA)

9. Schütze M, Campisanob A, Colas H, Schillingd W, Vanrolleghem P (2004) Real time control of urban wastewater systems: where do we stand today? J Hydrol 299:335-348

10. Blanke M, Kinnaert M, Lunze J, Staroswiecki M (2016) Diagnosis and fault-tolerant control, 3rd edn. Springer, Berlin, Heidelberg

11. Camacho EF, Bordons C (2004) Model predictive control, 2nd edn. Springer, London

12. Maciejowski JM (2002) Predictive control with constraints. Prentice Hall, Essex, England

13. Mays LW (2004) Urban stormwater management tools. McGrawHill Professional Publishing, USA

14. Cembrano G, Quevedo J, Salamero M, Puig V, Figueras J, Martí J (2004) Optimal control of urban drainage systems: a case study. Control Eng Pract 12(1):1-9

15. Ocampo-Martinez C, Puig V, Cembrano G, Creus R, Minoves M (2009) Improving water management efficiency by using optimization-based control strategies: the Barcelona case study. Water Sci Technol: Water Supply 9(5):565-575

16. Quevedo J, Puig V, Cembrano G, Blanch J (2010) Validation and reconstruction of flow meter data in the Barcelona water distribution network. Control Eng Pract 11(6):640-651

17. Quevedo J, Cembrano G, Valls A, Serra J (1998) Computer applications in water supply, volume I, chapter Time series modelling of water demand: a study on short-term and long-term predictions. Research Studies Press, Lechworth, England, pp 268-288

18. Ocampo-Martinez C, Fambrini V, Barcelli D, Puig V (2010) Model predictive control of drinking water networks: a hierarchical and decentralized approach. In: Proceedings of the American control conference, 2010, pp 3951-3956

19. Ocampo-Martinez C, Ingimundarson A, Puig V, Quevedo J (2008) Objective prioritization using lexicographic minimizers for MPC of sewer networks. IEEE Trans Control Syst Technol 16(1):113-121

20. Chen A, Xu X (2012) Goal programming approach to solving network design problem with multiple objectives and demand uncertainty. Expert Syst Appl 39(4):4160-4170

21. Toro R, Ocampo-Martinez C, Logist F, Van Impe J, Puig V (2011) Tuning of predictive controllers for drinking water networked systems. In: Proceedings of 18th IFAC world congress, Milano, Italy, August 2011, pp 14507-14512

22. Rawlings JB, Amrit R. Nonlinear model predictive control

23. Calafiore G, Dabbene F (2006) Probabilistic and randomized methods for design under uncertainty. Springer

24. Shapiro A, Dentcheva D, Ruszczynski A (2009) Lectures on stochastic programming: modeling and theory. Society for industrial and applied mathematics and mathematical programming society, 2009

25. Grosso JM. A robust adaptive model predictive control to enhance the management of drinkingwater networks subject to demand uncertainty and actuators degradation. Master's thesis, Technical University of Catalonia (UPC), Barcelona

26. Cembrano G, Quevedo J, Puig V, Perez R, Figueras J, Verdejo JM, Escaler I, Ramon G, Barnet G, Rodriguez P, Casas M (2011) PLIO: a generic tool for real-time operational predictive optimal control of water networks. Water Sci Technol 64(2):448-459 